\title{
Is video a useful means of sharing information? A Panel Discussion
}

\author{
D. C. A. Bulterman, Moderator \\ CWI: Centrum voor Wiskunde en Informatica \\ Amsterdam, The Netherlands \\ Dick.Bulterman@cwi.nl
}

\section{PANEL OVERVIEW}

One of the realities of the Web is that nearly everyone with a PC and a word processing package can act as an author and publisher, with the Web serving as a common, global distribution channel. One of the hopes of a next-generation Web is that nearly everyone with a video camera and a good idea will be able to become a movie producer or television star. Again, users provide content and "The Network" provides a means of sharing that content with the rest of the world.

How realistic is such a dream?

Use of the Web as an (expensive!) video player is not news. Many commercial products and protocols support the synchronized delivery of continuous media like audio and video. In order to do something useful with video, however, new facilities will be required that allow users to collect, edit and re-use video sequences in the same way that they can now collect, edit and re-use text and image data.

This panel will consider various issues associated with the creation and distribution of video data in a networked environment. We will look at:

- the viability of encoding schemes to contain meta-data that can be used to identify semantic content;

- the viability of locating a particular scene or shot from a move stored somewhere in cyberspace;

- the viability of creating a video using on-demand scatter-gather techniques;

- the viability of re-using content from broadcast sources that would otherwise stay on the cutting room floor.

In each case, panelists will comment on various scenarios for integrating video as part of the standard information stream.

The goal of the panel is to understand the issues and the time-frames involved in making continuous media an active communications mechanism, rather than a passive form of information or entertainment. 